\title{
4D Sommerfeld quantization of the complex extended charge
}

\author{
Igor E. Bulyzhenkov ${ }^{1,2, a}$ \\ ${ }^{1}$ Moscow Institute of Physics and Technology, 9 Institutskiy per., Dolgoprudny, Moscow reg., 141700, Russia \\ ${ }^{2}$ Lebedev Physics Institute RAS, 53 Lininsky pros., Moscow, 119991, Russia
}

\begin{abstract}
Gravitational fields and accelerations cannot change quantized magnetic flux in closed line contours due to flat 3D section of curved 4D space-time-matter. The relativistic Bohr-Sommerfeld quantization of the imaginary charge reveals an electric analog of the Compton length, which can introduce quantitatively the fine structure constant and the Plank length.
\end{abstract}

\section{London superfluid motion}

In 1939 Einstein first inferred the logical failure of the Schwarzschild curved metric with singularities for physical reality from a gedanken experiment [1]. Gravitation or acceleration analogs of the Aharonov-Bohm Effect were never found in SQUID experiments that indirectly confirms flatness of 3D space. Strict quantization of magnetic flux and steady SQUID measurements in time-varying gravitational and acceleration fields have refuted non-Euclidean metric approaches to 3D geometry in advanced metric theories of gravitational fields.

The superfluid motion of Cooper pairs in electromagnetic fields A can be formally described by the macroscopic Schrodinger equation with a complex continuous function $\Psi=n^{1 / 2} \exp (i \chi / \hbar c)$. This approach of condensed matter theory results in the well-tested London supercurrent,

$$
\mathbf{j} \equiv \frac{i \hbar q}{2 m}\left(\Psi^{*} \nabla \Psi-\Psi \nabla \Psi^{*}\right)+\frac{q^{2}}{2 m c}|\Psi|^{2} \mathbf{A}=\frac{q n}{m c}(\nabla \chi-q \mathbf{A})
$$

Reffered by London's 3-currents, we start 4-vector operations with the canonical four-momentum density, $n(x) P_{\mu}(x) \equiv-n(x) \nabla_{\mu} \chi / c=-n(x) \partial_{\mu} \chi / c$, of superelectrons using the phase function gradient $\nabla_{\mu} \chi$ and the covariant derivative $\nabla_{\mu}$ in metric space-time, $\mu \rightarrow\{0,1,2,3\}$. The symmetrical Christoffel coefficients in Einstein's General Relativity (GR) provide the universal covariant equality $\nabla_{\mu} P_{v}-$ $\nabla_{\nu} P_{\mu} \equiv \partial_{\mu} P_{\nu}-\partial_{\nu} P_{\mu} \equiv-\left(\partial_{\mu} \partial_{\nu}-\partial_{\nu} \partial_{\mu}\right) \chi \equiv 0$ for charged elementary matter independently from its density function $n(x)$. This material density, as was assumed by London in 1934, is to be finite in all space points where the single-valued phase $\chi$ of superfluid matter is defined.

\footnotetext{
ae-mail: bulyzhenkov.ie@ mipt.ru
} 


\section{4D path quantization of extended charged particles}

One may conventionally associate canonical energy-momentum densities of a distributed Cooper pair with relativistic gravitational and electromagnetic fields,

$$
-\frac{n}{c} \partial_{\mu} \chi=n g_{\mu \nu}\left(m c \frac{d x^{v}}{d s}+\frac{q}{c} A^{v}\right) \equiv n\left\{P_{o}, P_{i}\right\} \equiv n\left\{\frac{m c \sqrt{g_{o o}}}{\sqrt{1-v^{2} c^{-2}}}+\frac{g_{o v} q A^{v}}{c}, \frac{-m\left(\gamma_{i j} v^{j}+\sqrt{g_{o o}} g_{i} c\right)}{\sqrt{1-v^{2} c^{-2}}}+\frac{g_{i \nu} q A^{v}}{c}\right\},
$$

where $v^{i} \equiv c d x^{i}\left(g_{o o}\right)^{-1 / 2}\left(d x^{o}-g_{i} d x^{i}\right)^{-1} \equiv d x^{i} / d \tau$ is the relativistic three-velocity of the local analytical density $n=n(x)$ of the continuous charge $q=\int q n \sqrt{\left|\operatorname{det} \gamma_{i j}\right|} d V$ and the continuous mass $m=\int m n \sqrt{\left|\operatorname{det} \gamma_{i j}\right|} d V, v^{2} \equiv \gamma_{i j} v^{i} v^{j}, \gamma_{i j} \equiv g_{i} g_{j} g_{o o}-g_{i j}, g_{i} \equiv-g_{o i} / g_{o o}, i \rightarrow\{1,2,3\}$.

Now one can generalize the Bohr-Sommerfeld quantization rule on the self-coherent fourmomentum densities (2) of a distributed elementary particle with continuous mass, $m n(x) \sqrt{\left|\operatorname{det} \gamma_{i j}\right|}$, and charge, $q n(x) \sqrt{\left|\operatorname{det} \gamma_{i j}\right|}$, densities by taking into account the GR time synchronization, $d \tau \equiv$ $\sqrt{g_{o o}}\left(d x^{o}-g_{i} d x^{i}\right) / c=0$ or $d x^{o}=g_{i} d x^{i}$, for neighboring space points of closed line contours,

$$
\begin{aligned}
\pm 2 \pi \hbar N & =\frac{1}{c} \oint d x^{\mu} \nabla_{\mu} x_{s} \equiv \oint\left[-P_{i} d x^{i}-P_{o} d x^{o}\right] \equiv \oint_{d \tau=o}\left[\frac{m\left(\gamma_{i j} v^{j}+c \sqrt{g_{o o}} g_{i}\right)}{\sqrt{1-v^{2} c^{-2}}}-\frac{q\left(g_{i o} A^{o}+g_{i j} A^{j}\right)}{c}\right] d x^{i} \\
& -\oint_{d \tau=o}\left(\frac{m c \sqrt{g_{o o}}}{\sqrt{1-v^{2} c^{-2}}}+\frac{q\left(g_{o o} A^{o}+g_{o j} A^{j}\right)}{c}\right) g_{i} d x^{i} \equiv \oint_{d \tau=o}\left[\frac{m v^{j}}{\sqrt{1-v^{2} c^{-2}}}+\frac{q A^{j}}{c}\right] \gamma_{i j} d x^{i} .
\end{aligned}
$$

We arrived for the curved space-time to the very Bohr-Sommerfeld quantisation rule from the Special Theory of Relativity. The electric potential $A^{o}=A^{o}(x)$ is not relevant to the mechano-magnetic fluxoid quantization (3) for the charged elementary mass in electromagnetic and gravitational fields. Indeed, Coulomb electric fields $A^{o}(x)$ of external charges have never destroyed quantization of any elementary particle, including the Cooper boson of two superelectrons. Similarly, Newton gravitational field, or $g_{o o}(x)$, should also be irrelevant in practice to quantization of elementary matter. It is not surprising that this metric component has vanished in (3) for the relativistic contour quantization in 4D space-time.

On the other side, the 4D quantization rule (3) still corresponds to gravity-dependent 3D space with the inhomogeneous metric tensor $\gamma_{i j}(x)$. But gravity-dependent phase shifts along different path lines between two material points of the same continuous Cooper boson, for example, contradict to a single valued phase requirement for always self-coherent states of free elementary matter. Consequently, such path-varying shifts would destroy steady Bohr-Sommerfeld quantization in closed line contours. The observed magnetic flux quantization in superconducting loops has confirmed, in fact, strict spatial flatness of quantum reality, where $\gamma_{i j}(x)=\delta_{i j}$.

\section{Complex charge reveals meaning of $\alpha=1 / 137$ and $l_{p}=1.62 \times 10^{-35} \mathrm{~m}$}

The curved 3D metric solution was adopted in 1916 due to the use of the unrealistic empty space paradigm and the Newton point particle references rather than the continuous particle references from the 1925 nondual wave mechanics. Nowadays Einstein's metric formalism may replace the operator material density $\delta\left(\mathbf{x}-\mathbf{x}_{e}\right)$ of the postulated point particle with an analytical density of the continuous particle from the non-empty space paradigm. Author can propose, for instance, the elementary density solution $n_{e}\left(\mathbf{x}, \mathbf{x}_{e}, t\right)=r_{o} / 4 \pi\left(\mathbf{x}-\mathbf{x}_{e}\right)^{2}\left(r_{o}+\left|\mathbf{x}-\mathbf{x}_{e}\right|\right)^{2}$ to both Maxwell and Einstein equations with extended radial electron. The theory parameter $r_{o}=Q \sqrt{G} / c^{2}$ for electron's radial density in the classical (probability-free) is defined by the elementary charge $Q=\left(i e+\sqrt{G} m_{o}\right)$ having the center of spherical symmetry at $\mathbf{x}_{e}[2]$. 
Under such a non-empty-space approach, all metric components $g_{\mu v}$ of the strongly warped 4D space-time interval for distributed radial matter can depend on gravitational fields, but keep six inherent metric bounds or symmetries $g_{o i} g_{o j} g_{o o}^{-1}(x)=g_{i j}(x)+\delta_{i j}$. Pseudo-Riemannian 4-geometry with 6 inherent metric symmetries $\gamma_{i j}(x)=\delta_{i j}$ has the universal, Euclidean 3D space sub-interval in nonlocal GR equations and provides new physical interpretation of the known gravitational tests [3]. Strict spatial flatness of the warped space-time manifold reinforces the surface independent magnetic flux (3) over a closed line, the gauss electric flux over a closed surface, and gravity/inertia independent quantization of elementary matter in covariant relativistic relations.

Despite the nonlocal nature of radial sources in non-empty material space, the continuous electron behaves in collisions with energy exchanges like a material point, because half of electron's mass and charge is concentrated within the ultra small radius $l_{1 / 2}=\left|r_{o}\right|=1.38 \times 10^{-36} \mathrm{~m}$ (which is far behind the top resolution $10^{-19} \mathrm{~m}$ of space measurements in CERN).

Electron's relativistic 4-current $Q n_{e}\left(\mathbf{x}, \mathbf{x}_{e}, t\right) u_{\mu}$ is proportional to the complex energy 4-flow, $\varphi_{o} Q n_{e}(\mathbf{x}, t) u_{\mu}$, where $Q=\left(i e+\sqrt{G} m_{o}\right)$ is the complex charge of the elementary energy carrier and $\varphi_{o}=c^{2} / \sqrt{G}=1.04 \times 10^{27} V$ is the universal self-potential for the mass and the electric charge. Complex self- energy $E\left(\mathbf{x}_{e}\right)$ of such a distributed charge centered around $\mathbf{x}_{e}$ can be found from the volume integral

$$
E\left(\mathbf{x}_{e}\right)=\int d^{3} x \varphi_{o} Q n_{e}\left(\mathbf{x}, \mathbf{x}_{e}, t\right) u_{\mu} u^{\mu}=\varphi_{o} Q=m_{o} c^{2}+i e c^{2} G^{-1 / 2}=511 K e V+i 1.04 \times 10^{27} \mathrm{KeV} .
$$

The action of this complex elementary energy is given by the line integral over the 4-path $x_{e}^{\mu}$

$$
S=-\int \frac{\varphi_{o} Q u_{e \mu} d x_{e}^{\mu}}{c} \Rightarrow\left(m_{o} c+\frac{i e c}{\sqrt{G}}\right) \oint_{\tau=0} \sqrt{d \tau^{2}-d l^{2}}= \pm n h \pm i k h .
$$

From here one can introduce as the well known Compton length, $\bar{l}_{C}=\hbar / m_{o} c=3.86 \times 10^{-13} \mathrm{~m}$, as well as its electrical analog for the electron, $\bar{l}_{e}=\hbar \sqrt{G} / e c=1.89 \times 10^{-34} \mathrm{~m}$. By compare this fundamental length with the half-charge length $l_{1 / 2}$ of the radial distribution,

$$
\left\{\begin{array}{l}
\bar{l}_{e} / l_{1 / 2}=\hbar c / e^{2} \equiv \alpha^{-1}=137 \\
\bar{l}_{e} \cdot l_{1 / 2}=\hbar c / \varphi_{o}^{2}=\hbar G / c^{3} \equiv l_{p}^{2}=\left(1,62 \times 10^{-35} \mathrm{~m}\right)^{2} .
\end{array}\right.
$$

one can knowledgeable introduce into the extended electron theory the fine structure constant $\alpha$ and the Plank length $l_{P}$.

\section{References}

[1] A. Einstein, Annals of Mathematics, 40, 922 (1939).

[2] I. E. Bulyzhenkov, Complex Charge Densities Unify Particles with Fields and Gravitation with Electricity, Bullet. Lebedev Phys. Inst, 43, 140 (2016).

[3] I. E. Bulyzhenkov, Geometrization of radial particles in non-empty space complies with tests of General Relativity, Journal of Modern Physics, 3, N10, 1465 (2012). 